\title{
ON THE DIVISIBLE PARTS OF QUOTIENT GROUPS
}

\author{
Andreas Blass
}

\begin{abstract}
We study the possible cardinalities of the divisible part of $G / K$ when the cardinality of $K$ is known and when, for all countable subgroups $C$ of $G$, the divisible part of $G / C$ is countable.
\end{abstract}

\section{INTRODUCTION}

Let $G$ be an abelian group that is reduced, i.e., its divisible part $\operatorname{Div}(G)$ is zero. A quotient group $G / K$ need not be reduced. This paper is about the question how big $\operatorname{Div}(G / K)$ can be, relative to the cardinality $|K|$ of $K$.

John Irwin has suggested, as a natural weakening of freeness, the concept of a fully starred torsion-free group, i.e., a torsion-free, abelian group $G$ such that, for all subgroups $K,|\operatorname{Div}(G / K)| \leq|K|$. He asked whether, to test whether $G$ is fully starred, it suffices to check the definition for countable $K$. At first sight, this seems unlikely; how should the quotients by countable subgroups influence the quotients by larger subgroups. We shall show, however, that the answer to Irwin's question is affirmative if $|K|<\aleph_{\omega}$. We shall also show that it is consistent with the usual axioms of set theory (ZFC, i.e., Zermelo-Fraenkel set theory, including the axiom of choice) that the answer is negative for $|K|=\aleph_{\omega}$ but positive for many larger values of $|K|$. If a certain very large cardinal hypothesis is consistent, then it is also consistent that the answer to Irwin's question is affirmative for $|K|=\aleph_{\omega}$. It remains an open problem whether an affirmative answer, for all groups regardless of cardinality, is consistent (relative to some large cardinals).

The following table summarizes the results. The middle column gives what can be said about $|\operatorname{Div}(G / K)|$ when $|K|$ is as in the first column, when $\operatorname{Div}(G / C)$ is countable for all countable $C \leq G$, and when the set-theoretic hypothesis in the right column is satisfied. (The assumption about countable $C$ is irrelevant in the first, very elementary line of the table.)

1991 Mathematics Subject Classification. 20K20, 03E05, 03E35, 03E55, 03E75.

Partially supported by NSF grant DMS-9204276 and NATO grant LG921395.

This paper is in final form, and no version of it will be submitted for publication elsewhere. 


\begin{tabular}{|c|c|c|}
\hline$|K|=\kappa$ & $|\operatorname{Div}(G / K)|$ & Hypothesis \\
\hline Arbitrary & $\leq \kappa^{\aleph_{0}}$ & None \\
$\kappa<\aleph_{\omega}$ & $\leq \kappa$ & None \\
$\operatorname{cf}(\kappa) \neq \omega$ & $\leq \kappa$ & No inner model with measurable cardinal \\
$\operatorname{cf}(\kappa)=\omega$ & $\leq \kappa^{+}$ & No inner model with measurable cardinal \\
$\aleph_{\omega}$ & $\leq \aleph_{\omega}$ & Chang's conjecture for $\left(\aleph_{\omega+1}, \aleph_{\omega}\right)$ \\
$\aleph_{\omega}$ & possibly $=\aleph_{\omega+1}$ & $V=L$ \\
$\aleph_{\omega}$ & $<\aleph_{\aleph_{4}}$ & None \\
\hline
\end{tabular}

Some of the hypotheses in the right column can be weakened. See Theorems 10 and 12 for sharper statements.

\section{BACKGROUND}

Throughout this paper, all groups are abelian and reduced.

In this section, we present, for motivation and orientation, some elementary results and examples. No novelty is claimed for any of this material.

As Irwin pointed out when he suggested the study of fully starred torsion-free groups, this class of groups includes all free groups. Indeed, if $G$ is freely generated by a basis $B$ and if $K$ is a subgroup of $G$, then by expressing each element of $K$ as a combination of (finitely many) elements of $B$, we obtain a subset $B_{0}$ of $B$, no larger than $K$ in cardinality, such that $K$ is included in the subgroup $G_{0}$ of $G$ generated by $B_{0}$. Then $G / K \cong\left(G_{0} / K\right) \oplus F$ where $F$ is freely generated by $B-B_{0}$. Therefore the divisible part of $G / K$ coincides with that of $G_{0} / K$, whose cardinality is at most that of $G_{0}$, which equals that of $K$.

For non-free $G$, on the other hand, the divisible part of $G / K$ may well be larger than $K$. For example, if $G$ is the product of countably many infinite cyclic groups and $K$ is their direct sum (embedded in the product in the obvious way), then the divisible part of $G / K$ is easily seen to have the cardinality of the continuum, even though $K$ is countable. (Notice that, if the continuum hypothesis holds, then the group $G$ in this example is almost free in the sense that all subgroups of smaller cardinality are free [10].) Another example is obtained by taking $G$ to be the additive group of $p$-adic integers and $K$ the subgroup of ordinary integers; then $G / K$ has the cardinality of the continuum and is divisible.

For countable $K$, the preceding examples achieve the largest possible cardinality for $\operatorname{Div}(G / K)$, as the following proposition shows.

Proposition 1. For any (reduced) group $G$ and any subgroup $K$, we have that $|\operatorname{Div}(G / K)| \leq|K|^{\aleph_{0}}$.

Proof. Let $D$ be the pre-image of $\operatorname{Div}(G / K)$ in $G$, so $K \subseteq D \subseteq G$ and $D / K$ is divisible. We shall prove the proposition by producing a one-to-one function from $D$ into the set of countable sequences of elements of $K$. As $D / K$ is divisible, we have, for each $d \in D$ and each positive integer $n$, some $a_{n}(d) \in D$ and some $b_{n}(d) \in K$ such that $d=n \cdot a_{n}(d)+b_{n}(d)$. If $G$ is torsion-free, then the map we seek sends each $d \in D$ to the sequence $\left(b_{n}(d)\right)_{n \in \mathbb{N}}$. To see that it is one-to-one, 
$d-d^{\prime}=n \cdot\left(a_{n}(d)-a_{n}\left(d^{\prime}\right)\right)$. Thus, $d-d^{\prime}$ belongs to the divisible part of $G$ (here we use that $G$ is torsion-free), which is zero as $G$ is reduced.

To avoid the assumption that $G$ is torsion-free, it suffices to include, in the sequence associated to $d$, not only all the $b_{n}(d)$ but also all $b_{n}\left(a_{k}(d)\right)$, all $b_{n}\left(a_{k}\left(a_{l}(d)\right)\right)$, etc. Now, if $d$ and $d^{\prime}$ give rise to the same sequence, then the subgroup of $G$ generated by $d-d^{\prime}$ and all elements of the forms $a_{k}(d)-a_{k}\left(d^{\prime}\right), a_{k}\left(a_{l}(d)\right)-a_{k}\left(a_{l}\left(d^{\prime}\right)\right)$, etc. is divisible and therefore zero.

The proposition justifies the first line of the table in the introduction. As indicated there, this result, unlike the later ones, does not depend on any assumption about $\operatorname{Div}(G / C)$ for countable $C$, but only on the assumption (obviously needed) that $G$ is reduced.

We note that, by results of Cohen [2] and Solovay [9], the upper bound given by Proposition 1 is rather weak. Even when $K$ is countable, the bound $|K|^{\aleph_{0}}$, which then equals the cardinality of the continuum, can be an arbitrarily large cardinal.

\section{Some Combinatorial Set Theory}

We follow the usual set-theoretic conventions whereby an ordinal number is the set of all smaller ordinal numbers and a cardinal number is the first ordinal of that cardinality. In particular, the cardinal $\aleph_{0}$ is identified with the first infinite ordinal number $\omega$ and with the set of all natural numbers.

For any set $A$, let $[A]^{\omega}$ be the set of all countably infinite subsets of $A$, partially ordered by the subset relation. We shall need some information about the cofinality $\operatorname{cf}\left([A]^{\omega}\right)$, i.e., the smallest possible size for a family of countable subsets of $A$ such that every countable subset of $A$ is included in one from that family. Of course this depends only on the cardinality of $A$, so we may assume that $A$ is a cardinal. The following proposition summarizes the information we need about $\operatorname{cf}\left([\kappa]^{\omega}\right)$ for uncountable $\kappa$; it is taken from Section 4 of [6]. The hypothesis "the covering lemma over a model of GCH" that occurs in the second part of the proposition means that there is a class $M$ of sets such that every member of a member of $M$ is in $M$ (one says $M$ is transitive), all axioms of ZFC and the generalized continuum hypothesis (GCH) hold in $M$, and every uncountable set of ordinal numbers is included in a set of the same cardinality that is a member of $M$. We shall never need to use this definition of the covering lemma over a model of $\mathrm{GCH}$, but it is relevant that this assumption is satisfied unless there are inner models with measurable cardinals [3,4], i.e., transitive classes $M$ such that all axioms of ZFC and the statement "a measurable cardinal exists" hold in $M$. In fact, it is known that if this assumption fails then there are inner models with far stronger large cardinal properties than just a measurable cardinal; see for example [8].

Proposition 2. The equation $c f\left([\kappa]^{\omega}\right)=\kappa$ holds for all uncountable cardinals $\kappa<$ $\aleph_{\omega}$. If the covering lemma holds over a model of $G C H$, then the same equation holds for all cardinals $\kappa$ of uncountable cofinality, while for uncountable $\kappa$ of countable cofinality $c f\left([\kappa]^{\omega}\right)=\kappa^{+}$.

Proof. See [6], Corollary 4.8, Lemma 4.10, and the proof of the latter.

We note that the result obtained for the case of countable cofinality using the covering lemma is optimal; that is, for $\operatorname{cf}(\kappa)=\omega$, we cannot have $\operatorname{cf}\left([\kappa]^{\omega}\right)=\kappa$. Indeed, given a family $\mathcal{F}$ of $\kappa$ countable subsets of $\kappa$ and given an increasing $\omega$ - 
$\mathcal{F}_{i}$ of respective cardinalities $\theta_{i}$, and we can find for each $i$ an element $x_{i} \in \theta_{i+1}$ not in the union of $\mathcal{F}_{i}$ (for this union has size at most $\theta_{i} \cdot \aleph_{0}$ ). Then the countable set consisting of these $x_{i}$ 's is not included in any member of $\mathcal{F}$.

In the absence of the covering lemma, it is much more difficult to bound $\operatorname{cf}\left([\kappa]^{\omega}\right)$ for $\kappa$ of cofinality $\omega$. The following proposition is one of the surprising results of Shelah's recently developed theory of possible cofinalities. It is stated (with a hint about the proof) as Proposition 7.13 of [1], attributed to Baumgartner.

Proposition 3. $c f\left(\left[\aleph_{\omega}\right]^{\omega}\right)<\aleph_{\aleph_{4}}$.

The remainder of this section is devoted to a combinatorial principle closely related to the preceding cofinality considerations but, as we shall see, of more direct relevance to the size of the divisible parts of quotient groups.

For infinite cardinals $\kappa<\lambda$, we say that the $\lambda$ to $\kappa$ compression principle holds and we write $\operatorname{CP}(\lambda \rightarrow \kappa)$ if, for any $\lambda$-indexed family $\left(X_{i}\right)_{i \in \lambda}$ of countable subsets of $\kappa$, there is an uncountable set of indices, $Z \subseteq \lambda$, such that $\bigcup_{i \in Z} X_{i}$ is countable. Intuitively, this means that, if $\lambda$ countable sets are packed (with overlapping) into a set of size $\kappa$ (non-trivial packing, as $\kappa<\lambda$ ), then some uncountably many of those sets must have been packed into a countable set (so we have non-trivial packing on a smaller scale).

There is a trivial connection between the compression principle and the cofinalities considered above; we state it as a proposition for future reference.

Proposition 4. If $\lambda>c f\left([\kappa]^{\omega}\right)$ then $C P(\lambda \rightarrow \kappa)$ holds.

Proof. Fix a family $\mathcal{F}$ of strictly fewer than $\lambda$ countable subsets of $\kappa$ cofinal in $[\kappa]^{\omega}$, and fix $\lambda$ countable subsets $X_{i}$ of $\kappa$. Each $X_{i}$ is in some member of $\mathcal{F}$. As there are $\lambda X_{i}$ 's and fewer members of $\mathcal{F}$, uncountably many $X_{i}$ 's (in fact $\lambda$ of them) must be included in a single element of $\mathcal{F}$ and must therefore have a countable union.

The converse of Proposition 4 is not provable, at least if sufficiently large cardinals are consistent. The counterexample involves Chang's conjecture. Originally, Chang's conjecture was that, for a countable first-order language, every structure of cardinality $\aleph_{2}$ in which a particular unary predicate symbol $P$ denotes a set of cardinality $\aleph_{1}$ has an elementary submodel of cardinality $\aleph_{1}$ in which $P$ denotes a set of cardinality $\aleph_{0}$. Generalizing this by changing $\aleph_{2}$ and $\aleph_{1}$ in the hypothesis to $\lambda$ and $\kappa$, respectively, but leaving the conclusion unchanged, one has the Chang conjecture for $(\lambda, \kappa)$. The conjecture can be restated in a form, more convenient for our purposes, that avoids model-theoretic notions. We adopt this restatement as the definition: Chang's conjecture for $(\lambda, \kappa)$ is the assertion that, for any countably many functions $f_{n}$, each mapping some finite power $\lambda^{p}$ of $\lambda$ into $\kappa$ (where $p$ can depend on $n$ ), there is an uncountable subset $H$ of $\lambda$ such that each $f_{n}$ restricted to $H$ (more precisely, to $H^{p}$ ) has countable range. We shall need an elementary connection between Chang's conjecture and the compression principle and a (nonelementary) theorem from [7] giving the consistency of a particular instance of Chang's conjecture.

Proposition 5. Chang's conjecture for $(\lambda, \kappa)$ implies $C P(\lambda \rightarrow \kappa)$.

Proof. Assume Chang's conjecture for $(\lambda, \kappa)$, and let $\lambda$ countable subsets $X_{i}, i<\lambda$ of $\kappa$ be given. Fix, for each $i$ an enumeration of $X_{i}$ by natural numbers, and 
chosen enumeration of $X_{i}$. By Chang's conjecture for $(\lambda, \kappa)$, there is an uncountable $Z \subseteq \lambda$ whose images under all the $f_{n}$ are countable. Then $\bigcup_{i \in Z} X_{i}$, which is also the union of these countably many countable images, is countable, as required by the compression principle.

To state concisely the consistency theorem for Chang's conjecture for $(\lambda, \kappa)=$ $\left(\aleph_{\omega+1}, \aleph_{\omega}\right)$, we introduce a short name for the large cardinal hypothesis needed. (We shall not explicitly use this hypothesis, so the reader can safely skip the definition and remember only that we are dealing with very large cardinals, much larger than measurable cardinals but not known or even widely believed to be inconsistent.) A cardinal $\kappa$ will be called huge + if there is an elementary embedding $j$ of the settheoretic universe into a transitive class $M$ such that $\kappa$ is the first ordinal moved and, if $\mu$ denotes the $(\omega+1)$ th cardinal after $j(\kappa)$ then every function from $\mu$ into $M$ is an element of $M$. (Huge is defined the same way except that $\mu=j(\kappa)$.)

Proposition 6. If the existence of a huge+ cardinal is consistent with ZFC, then so is Chang's conjecture for $\left(\aleph_{\omega+1}, \aleph_{\omega}\right)$.

Proof. See Levinski, Magidor, and Shelah [7], Theorem 5.

We conclude this section with an analog for the compression property of a wellknown fact about $\operatorname{cf}\left([\kappa]^{\omega}\right)[6]$, Lemma 4.6.

Proposition 7. If $\lambda>\kappa^{+}$and if $C P(\lambda \rightarrow \kappa)$ then $C P\left(\lambda \rightarrow \kappa^{+}\right)$. More generally, if $\lambda>\mu$, if $\operatorname{cf}(\mu)>\omega$, and if $C P(\lambda \rightarrow \kappa)$ for all $\kappa<\mu$, then $C P(\lambda \rightarrow \mu)$.

Proof. Since $\operatorname{CP}(\lambda \rightarrow \kappa)$ trivially implies $\mathrm{CP}(\lambda \rightarrow \theta)$ for all $\theta<\kappa$, it suffices to prove the second statement. Let $\lambda$ countable subsets $X_{i}$ of $\mu$ be given. As $\operatorname{cf}(\mu)>$ $\omega$, each $X_{i}$ has its supremum $<\mu$, and, as $\lambda>\mu$, this supremum must be the same ordinal, say $\alpha$, for $\lambda$ of the $X_{i}$ 's. But then $\operatorname{CP}(\lambda \rightarrow|\alpha|)$ ensures that some uncountably many of these $X_{i}$ have a countable union.

\section{Divisible Parts of Quotients Are Not Too Large}

In this section, we present our positive results about Irwin's question described in the introduction. That is, we assume that $|\operatorname{Div}(G / C)|$ is countable for all countable subgroups $C$ of $G$, and we deduce upper bounds for $|\operatorname{Div}(G / K)|$ in terms of $|K|$. All these results are obtained by combining the set-theoretic results in Section 2 with the following proposition which relates the divisible parts of quotient groups to the compression principle.

Proposition 8. Let $G$ be a group such that Div $(G / C)$ is countable for all countable subgroups $C$ of $G$. Let $\lambda>\kappa$ be cardinals such that $C P(\lambda \rightarrow \kappa)$ holds. Then for all subgroups $K$ of $G$ of cardinality $\kappa,|\operatorname{Div}(G / K)|<\lambda$.

Proof. Assume toward a contradiction that the hypotheses hold but the conclusion fails. Then there is a subgroup $D$ of cardinality $\lambda$ with $K \subseteq D \subseteq G$ and with $D / K$ divisible. As in the proof of Proposition 1, associate to each $d \in D$ and each positive integer $n$ elements $a_{n}(d) \in D$ and $b_{n}(d) \in K$ such that $d=n \cdot a_{n}(d)+b_{n}(d)$. For each $d \in D$, let $X_{d}$ be the set of those elements of $K$ obtainable by applying to $d$ any finite composite of the functions $a_{n}: D \rightarrow D$ followed by any of the functions $b_{n}: D \rightarrow K$. (The identity function counts as a finite composite of $a_{n}$ 's, namely the composite of none.) Thus $X_{d}$ consists of all elements of the forms $b_{n}(d), b_{n}\left(a_{k}(d)\right)$, 
As each $X_{d}$ is a countable subset of the $\kappa$-element set $K$, the assumed $\lambda$ to $\kappa$ compression principle provides an uncountable subset $Z$ of $D$ such that all $X_{d}$ for $d \in Z$ are contained in a countable subset $C$ of $K$. We may assume that $C$ is a subgroup of $K$, by replacing it with the subgroup it generates.

Let $E$ be the subgroup of $D$ generated by all the elements obtainable from elements of $Z$ by applying any finite composite of the functions $a_{n}$. Notice that, if $e$ is any one of these generators, then $b_{n}(e) \in C$ and $a_{n}(e) \in E$ for all positive integers $n$. Thus, from $e=n \cdot a_{n}(e)+b_{n}(e)$, we infer that $E / C$ is divisible. But, as $E$ is uncountable (containing $Z$ ) and $C$ is countable, $E / C$ is an uncountable subgroup of $G / C$, contrary to the assumption that, for countable $C, \operatorname{Div}(G / C)$ is countable.

Corollary 9. Let $G$ be a group such that Div $(G / C)$ is countable for all countable subgroups $C$ of $G$. If $K$ is a subgroup of $G$ of cardinality $\kappa$, then $|\operatorname{Div}(G / K)| \leq$ $c f\left([\kappa]^{\omega}\right)$.

Proof. Combine Propositions 4 and 8.

Theorem 10. Let $G$ be a group such that Div $(G / C)$ is countable for all countable subgroups $C$ of $G$, and let $K$ be a subgroup of $G$ of cardinality $\kappa$.

(1) If $\kappa<\aleph_{\omega}$ then $|\operatorname{Div}(G / K)| \leq \kappa$.

(2) If the covering lemma holds over a model of GCH (in particular if there is no inner model with a measurable cardinal) and if $c f(\kappa)>\omega$, then $|\operatorname{Div}(G / K)| \leq \kappa$.

(3) If the covering lemma holds over a model of GCH (in particular if there is no inner model with a measurable cardinal) and if $c f(\kappa)=\omega$, then $|\operatorname{Div}(G / K)| \leq \kappa^{+}$.

(4) If Chang's conjecture for $\left(\aleph_{\omega+1}, \aleph_{\omega}\right)$ is true and if $\aleph_{\omega} \leq \kappa<\aleph_{\omega \cdot 2}$, then $|\operatorname{Div}(G / K)| \leq \kappa$.

(5) If $\kappa=\aleph_{\omega}$ then $|\operatorname{Div}(G / K)|<\aleph_{\aleph_{4}}$.

Proof. (1), (2), and (3) all follow immediately from Proposition 2 and Corollary 9. For (4), use Propositions 5 and 7 to deduce from Chang's conjecture for $\left(\aleph_{\omega+1}, \aleph_{\omega}\right)$ that $\mathrm{CP}(\kappa \rightarrow)+\kappa$ holds for all $\kappa$ as in (4); then invoke Proposition 8. (5) follows from Proposition 3 and Corollary 9.

It follows from (1), (4) and Proposition 6 that the statement "If $G$ is a group such that $\operatorname{Div}(G / C)$ is countable for all countable subgroups $C$ of $G$, and if $K$ is a subgroup of $G$ with $|K|<\aleph_{\omega \cdot 2}$, then $|\operatorname{Div}(G / K)| \leq|K|$ " is consistent relative to a huge+ cardinal.

The results presented in this section complete the justification of all lines in the table in the introduction except for the next to last line. The one remaining line, asserting that the answer to Irwin's question is negative for groups of cardinality $\aleph_{\omega}$ if $V=L$, is the subject of the next two sections.

\section{More Combinatorial Set Theory}

In this section, we develop, under the assumption $V=L$ the set theory needed to produce a counterexample for Irwin's question. In fact, we do not need the full 
cardinal $\kappa, \square_{\kappa}$ denotes the following assertion: There exists a sequence of sets $\left(C_{\xi}\right)$ indexed by the limit ordinals $\xi<\kappa^{+}$such that for each such $\xi$

(1) $C_{\xi}$ is a closed, cofinal subset of $\xi$.

(2) If $\operatorname{cf}(\xi)<\kappa$ then $\left|C_{\xi}\right|<\kappa$.

(3) If $\eta$ is a limit point of $C_{\xi}$, then $C_{\eta}=\eta \cap C_{\xi}$.

These square principles were introduced by Jensen [5] who showed that they follow from $V=L$ for all $\kappa$.

In order to obtain a negative answer to Irwin's question at cardinality $\aleph_{\omega}$, it is necessary, according to Proposition 8 , to violate $\mathrm{CP}\left(\aleph_{\omega+1} \rightarrow \aleph_{\omega}\right)$, i.e., to produce $\aleph_{\omega+1}$ countable subsets of $\aleph_{\omega}$ such that no countable set contains uncountably many of them. The following proposition shows that this and a bit more (which we shall need in the next section) can be done if $\square_{\aleph_{\omega}}$ holds. I am not sure to whom to attribute this proposition. Menachem Kojman told me that square aleph $_{\omega}$ contradicts $\mathrm{CP}\left(\aleph_{\omega+1} \rightarrow \aleph_{\omega}\right)$. Menachem Magidor showed me the proof given below for this fact and the additional information in Proposition 11. Magidor also informed me that similar arguments were known to Saharon Shelah.

Proposition 11. Assume $\square_{\aleph_{\omega}}$. There are $\aleph_{\omega+1}$ countably infinite subsets $X_{\xi}$ of $\aleph_{\omega}$ such that the intersection of every two of these $X_{i}$ is finite and, for each countable $Y \subseteq \aleph_{\omega}$, at most countably many of the $X_{\xi}$ have infinite intersection with $Y$ (and, a fortiori, at most countably many $X_{\xi}$ are included in $Y$ ).

Proof. Fix $C_{\xi}$ for limit ordinals $\xi<\aleph_{\omega+1}$ as in the definition of $\square_{\aleph_{\omega}}$. We shall define functions $f_{\xi}$ for $\xi<\aleph_{\omega+1}$ with the following properties.

(1) Each $f_{\xi}$ is a function on $\omega$ satisfying $f_{\xi}(n) \in \aleph_{n}$ for all $n \in \omega$.

(2) If $\xi<\eta$, then $f_{\xi}(n)<f_{\eta}(n)$ for all but finitely many $n \in \omega$.

(3) If $\eta$ is a limit ordinal, $\left|C_{\eta}\right|<\aleph_{n}$, and $\xi \in C_{\eta}$, then $f_{\xi}(n)<f_{\eta}(n)$.

(Notice that, for $\xi \in C_{\eta}$, (3) amplifies (2) by specifying that the finitely many exceptional $n$ in (2) are bounded above by the $q$ such that $\left|C_{\eta}\right|=\aleph_{q}$. Notice also that such a $q$ exists by (3) in the definition of $\square_{\aleph_{\omega}}$.) After constructing such $f_{\xi}$ 's, we shall show that their graphs are essentially the sets needed to establish the proposition.

The construction of the $f_{\xi}$ 's proceeds by induction on $\xi<\aleph_{\omega+1}$. Suppose, therefore, that $f_{\xi}$ is defined for every $\xi<\eta$, and we wish to define $f_{\eta}$.

If $\eta$ is not a limit ordinal, then (3) does not apply to $\eta$, so we need only satisfy (1) and (2), which we do as follows. Partition $\eta$ (the set of ordinals smaller than $\eta$ ), which has cardinality at most $\aleph_{\omega}$, into countably many pieces $A_{0}, A_{1}, \ldots$ such that each $A_{k}$ has cardinality at most $\aleph_{k}$. Then define $f_{\eta}(n)$ to be any ordinal that is $\left\langle\aleph_{n}\right.$ (so (1) holds) but $>f_{\xi}(n)$ for all $\xi \in A_{0} \cup \cdots \cup A_{n-1}$ (so that (2) holds, because if $\xi \in A_{p}$ then $f_{\xi}(n)<f_{\eta}(n)$ for all $\left.n>p\right)$. Such an ordinal exists, because there are only $\aleph_{n-1}$ ordinals $\xi \in A_{0} \cup \cdots \cup A_{n-1}$ and therefore the corresponding $f_{\xi}(n)$ 's cannot be cofinal in $\aleph_{n}$.

If $\eta$ is a limit ordinal, let $q$ be the natural number such that $\left|C_{\eta}\right|=\aleph_{q}$. Then, in order to satisfy (3), we must make sure that $f_{\eta}(n)$ satisfies, in addition to the requirements in the preceding paragraph, $f_{\xi}(n)<f_{\eta}(n)$ if $\xi \in C_{\eta}$ and $n>q$. But there are only $\aleph_{q}$ such $\xi$ 's, by choice of $q$, so the corresponding $f_{\xi}(n)$ 's cannot be cofinal in $\aleph_{n}$ when $n>q$. So an appropriate value for $f_{\eta}(n)$ can again be found. 
We claim that, from any uncountable $A \subseteq \aleph_{\omega+1}$, one can extract an uncountable $B \subseteq A$ and one can find $m \in \omega$ such that $f_{\xi}(n)<f_{\eta}(n)$ for all $\xi<\eta$ in $B$ and all $n \geq m$. (That is, the finitely many exceptions in (2) are bounded by the same $m$, independent of $\xi$ and $\eta$, as long as these two indices lie in $B$.) To prove this claim, consider an arbitrary uncountable $A \subseteq \aleph_{\omega+1}$. We may assume without loss of generality that $A$ has order type $\aleph_{1}$; just replace $A$ by the subset consisting of its first $\aleph_{1}$ elements. Let $\beta$ be the supremum of $A, \operatorname{so} \operatorname{cf}(\beta)=\aleph_{1}$. Inductively choose

$$
\gamma(0)<\alpha(0)<\gamma(1)<\alpha(1)<\cdots<\gamma(\omega)<\alpha(\omega)<\gamma(\omega+1)<\ldots
$$

for $\aleph_{1}$ steps, so that all the $\gamma$ 's are limit points of $C_{\beta}$ and all the $\alpha$ 's are in $A$. There is no difficulty making these choices, as both the set of limit points of $C_{\beta}$ and $A$ are cofinal in $\beta$. For each $\mu<\aleph_{1}$, (2) provides a natural number $m(\mu)$ such that

$$
\forall n \geq m(\mu) \quad f_{\gamma(\mu)}(n)<f_{\alpha(\mu)}(n)<f_{\gamma(\mu+1)}(n)
$$

Increasing $m(\mu)$ if necessary, we may assume that $\left|C_{\beta}\right|<\aleph_{m(\mu)}$. As there are uncountably many $\mu$ 's and only countably many possible values for $m(\mu)$, there is an uncountable $Y \subseteq \aleph_{1}$ such that $m(\mu)$ has the same value $m$ for all $\mu \in Y$. Now if $\mu<\nu$ are in $Y$ and if $n \geq m$ then we have

$$
f_{\alpha(\mu)}(n)<f_{\gamma(\mu+1)}(n) \leq f_{\gamma(\nu)}(n)<f_{\alpha(\nu)}(n)
$$

where the first and third inequalities hold because $n \geq m=m(\mu)=m(\nu)$, and the middle inequality holds because of (3) and the fact that, since the $\gamma$ 's are limit points of $C_{\beta}$, either $\mu+1=\nu$ or $\left.\gamma(\mu+1) \in \gamma(\nu) \cap C_{\beta}=C_{(} \nu\right)$. This means that $B=\{\alpha(\mu) \mid \mu \in Y\}$ and $m$ are as required in the claim.

Finally, we show that the functions $f_{\xi}$ (for all $\xi \in \aleph_{\omega+1}$ ), regarded as sets of ordered pairs $\subseteq \omega \times \aleph_{\omega}$, have the properties that every two have finite intersection and that no countable set $C$ can have infinite intersections with uncountably many $f_{\xi}$ 's. Then, transferring these subsets of $\omega \times \aleph_{\omega}$ to subsets of $\aleph_{\omega}$ by some bijection, we have the sets required by the proposition. That every two of the graphs have finite intersection is immediate from (2). To prove the other property, suppose $C$ were a countable set having infinite intersection with $f_{\xi}$ for all $\xi$ in some uncountable $A \subseteq \aleph_{\omega+1}$. Let $B \subseteq A$ and $m$ be as in the claim proved in the preceding paragraph. Then by deleting the first $m$ elements from each of the graphs $f_{\xi}, \xi \in B$, i.e., by forming $f_{\xi} \uparrow(\omega-m)$, we would obtain uncountably many pairwise disjoint sets, all intersecting the countable set $C$. As this is absurd, the proof is complete.

\section{A Quotient with a Large Divisible Part}

In this section we construct, assuming $\square_{\aleph_{\omega}}$, a counterexample to Irwin's question, i.e., a group $G$ such that $\operatorname{Div}(G / C)$ is countable for all countable $C$ but $G$ is not fully starred.

Theorem 12. Assume $\square_{\aleph_{\omega}}$. There exist a group $G$ of cardinality $\aleph_{\omega+1}$ and a subgroup $K$ of cardinality $\aleph_{\omega}$ such that $G / K$ is divisible but, for each countable subgroup $C$ of $G$, the divisible part of $G / C$ is countable.

Proof. The required groups $G$ and $K$ will actually be modules over the $\operatorname{ring} R$ of 
except 2. We write $R^{*}$ for the set of units of $R$, the rational numbers whose numerators and denominators (in reduced form) are both odd.

Since $\square_{\aleph_{\omega}}$ is assumed, let $X_{i}$ for $i \in \aleph_{\omega+1}$ be as in Proposition 11. Fix, for each $i$ a bijection between $X_{i}$ and the set $\mathbb{Z}$ of integers, and write $\xi(i, n)$ for the element of $X_{i}$ that corresponds to the integer $n$. (Notice that the same ordinal can be $\xi(i, n)$ for many different pairs $(i, n)$.)

The group $G$ will be presented as the $R$-module generated by certain objects subject to certain relations. The generators are of two sorts. First, there are $\aleph_{\omega}$ generators which, to simplify notation, we take to be the ordinals $\alpha<\aleph_{\omega}$. Second, there are $\aleph_{\omega+1}$ generators $g(i, n)$ indexed by all the ordinals $i<\aleph_{\omega+1}$ and all integers $n \in \mathbb{Z}$. The defining relations are

$$
g(i, n)=2 \cdot g(i, n+1)+\xi(i, n) .
$$

This defines $G$ as an $R$-module, hence as a group. $K$ is defined to be the submodule generated by the first sort of generators of $G$, the ordinals below $\aleph_{\omega}$.

Notice that, in any non-trivial $R$-linear combination of the defining relations of $G$, some $g(i, n)$ must occur. Indeed, of the finitely many $g(i, n)$ 's that occur in the relations being combined, the ones with the largest (or the smallest) values of $n$ cannot be canceled. So the relations impose no restrictions on the generators of $K$ alone. This means that $K$ is freely generated, as an $R$-module, by the ordinals $\alpha<\aleph_{\omega}$. It follows, exactly as in the argument for free groups in Section 1 , that $K$ is fully starred.

The quotient group $G / K$ can be presented by adjoining to the defining relations of $G$ the new relations $\alpha=0$ for all the generators $\alpha$ of $K$. The resulting presentation is clearly equivalent to one that has only the generators $\bar{g}(i, n)$ (where the bar over a letter denotes the coset modulo $K$ ) and the relations

$$
\bar{g}(i, n)=2 \cdot \bar{g}(i, n+1)
$$

Thus, $G / K$ is divisible and is in fact the rational vector space freely generated by the $\bar{g}(i, 0)$ 's; here $\bar{g}(i, n)$ is identified with $2^{-n} \bar{g}(i, 0)$.

To complete the proof of the theorem, it remains to show that $\operatorname{Div}(G / C)$ is countable for all countable subgroups $C$ of $G$. As a preliminary step toward this, we introduce a normal form for elements of $G$. We claim that every element $x$ of $G$ can be uniquely represented in the form

$$
x=\sum_{i} r_{i} g\left(i, n_{i}\right)+\sum_{\alpha} s_{\alpha} \alpha
$$

where all $r_{i} \in R^{*}, s_{\alpha} \in R$, and the summation variables $i$ and $\alpha$ range over some finite subsets (depending on $x$ ) of $\aleph_{\omega+1}$ and $\aleph_{\omega}$, respectively. (In particular, each $i$ that occurs at all, as the first argument of a $g$, occurs in only one term of such a normal form.) To produce such a normal form for $x$, we first write the image of $x$ in $G / K$ as a rational linear combination of the $\bar{g}(i, 0)$ 's, then we put the rational coefficients in this combination into $R^{*}$ by using $2^{n} \bar{g}(i, 0)=\bar{g}(i,-n)$, which holds in $G / K$, then we remove the bars from the $g$ 's in this expression, obtaining an element $y \in G$, and we observe that the difference $d=x-y$ is in $K$ as $y$ has the same image as $x$ in $G / K$. Now the desired normal form of $x$ consists of $y$ plus the 
by observing that the $\sum_{i} r_{i} g\left(i, n_{i}\right)$ part of a normal form of $x$ must have the same image as $x$ in $G / K$ and must therefore be exactly the $y$ constructed above.

It will be useful later to have a more computational description of how to convert an arbitrary element of $G$, given as an $R$-linear combination of generators $g(i, n)$ and $\alpha$, to normal form. This means that we must convert the given expression, using the defining relations of $G$, to one in which, for each $i$, there is at most one term of the form $r \cdot g(i, n)$ and the coefficient $r$ of any such term is in $R^{*}$. Suppose that, for a certain $i$, the given expression contains several terms of the form $g(i, n)$, possibly with different values of $n$. The defining relations of $G$ allow us to replace $g(i, n)$ with $g(i, n+1)+\xi(i, n)$, so we can increase the $n$ 's involved in these terms as much as we wish. In particular, we can increase them until they are all equal to, say, the largest of the originally occurring $n$ 's. At this stage, all the $g(i, n)$ 's, for the particular $i$ under consideration, have the same $n$, so we can collect them into one term by adding their coefficients. If the resulting coefficient of $g(i, n)$ is divisible by 2 , then we can use the defining relations "in reverse" to divide the coefficient by 2 while decreasing $n$ by 1 and subtracting $\xi(i, n-1)$. Repeat this until the coefficient is no longer divisible by 2 , i.e., until it is in $R^{*}$. By carrying out the procedure just described for each $i$, we clearly achieve normal form.

We point out two consequences of this procedure that will be useful later. First, notice that the element $\sum_{\alpha} s_{\alpha} \alpha \in K$ occurring in the normal form at the end consists of first the linear combination of $\alpha$ 's in the original expression, and second some terms of the form $\pm 2^{k} \xi(i, n)$, where the $i$ 's involved in these $\xi$ 's were involved in $g$ 's in the original expression.

Second, if $x-2 z \in K$ then $g(i, n)$ occurs in the normal form of $x$ if and only if $g(i, n+1)$ occurs in the normal form of $z$, and they occur with the same coefficient. To see this, start with a normal form of $z$, multiply all terms by 2 and add a suitable element of $K$ to get an expression for $x$ that is in normal form except that all the coefficients of $g$ 's are divisible once by 2 ; then apply the normalization algorithm to this expression.

We now embark on the proof that $\operatorname{Div}(G / C)$ is countable for all countable $C \subseteq G$. Notice first that, if $C$ were a counterexample and if $C^{\prime}$ were any countable group such that $C \subseteq C^{\prime} \subseteq G$, then $C^{\prime}$ would also be a counterexample. Indeed, there would be an uncountable $D$ with $C \subseteq D \subseteq G$ and $D / C$ divisible. Then, as $C \subseteq D \cap C^{\prime},\left(D+C^{\prime}\right) / C^{\prime} \cong D /\left(D \cap C^{\prime}\right)$ is a quotient of the divisible group $D / C$, and is therefore divisible. As $D+C^{\prime}$ is uncountable and $C^{\prime}$ is countable, we have another counterexample, as claimed.

Therefore, in proving that $G / C$ is divisible, we may assume that $C$ satisfies the following four conditions, since each condition amounts to being closed under countably many functions and can therefore be satisfied by a suitable countable supergroup of any given countable $C$.

(1) $C$ is an $R$-submodule of $G$.

(2) If $x \in C$, then all the generators $g(i, n)$ and $\alpha$ that occur in the normal form of $x$ are also in $C$.

(3) If $g(i, n) \in C$, then $g(i, m) \in C$ for all integers $m$.

(4) If $g(i, n) \in C$, then all members $\xi(i, m)$ of $X_{i}$ are in $C$.

We call $i<\aleph_{\omega+1}$ a $C$-index if, for some $n, g(i, n) \in C$. By (2), it is equivalent to say that $g(i, n)$ occurs in the normal form of some element of $C$, and by (3) it is 
only countably many $C$-indices. (Note, by contrast, that there may be uncountably many $i$ such that, for some $n, \xi(i, n) \in C$, for the same element of $C$ can be $\xi(i, n)$ for many different $i$.)

Now suppose $D$ is a subgroup of $G$ with $C \subseteq D$ and with $D / C$ divisible. We must prove that $D$ is countable. Call an element $i \in \aleph_{\omega+1}$ relevant if the normal form of some element of $D$ contains $g(i, n)$ for some $n$.

Lemma. Only countably many $i$ are relevant.

Proof. Suppose uncountably many $i$ are relevant. Then the set $U$ of relevant $i$ that are not $C$-indices is uncountable. As the $X_{i}$ were chosen as in Proposition 11, the countable set $C \cap \aleph_{\omega}$ (i.e., the set of generators of $K$ that are in $C$ ) must have finite intersection with $X_{i}$ for some $i \in U$. Fix such an $i$ for the rest of the proof of the lemma. Thus, $i$ is relevant, $i$ is not a $C$-index, and $C \cap X_{i}$ is finite.

As $i$ is relevant, choose an element $d_{0} \in D$ such that $g(i, q)$ occurs in $d_{0}$ for some $q$. (We think of $d_{0}$ and the other elements defined below as written in normal form, so "occurs in $d_{0}$ " really means "occurs in the normal form of $d_{0}$.") Let $a \in R^{*}$ be the coefficient of $g(i, q)$ in $d_{0}$. Let $J$ be the set of those $j$ such that (i) $g(j, m)$ occurs in $d_{0}$ for some $m$, (ii) $j \neq i$, and (iii) $j$ is not a $C$-index. Of course, $J$ is finite.

Since $X_{i}$ has finite intersection with $C$ (by choice of $i$ ) and with $X_{j}$ for each $j \in J$ (by the "finite pairwise intersections" part of Proposition 11), fix $p \in \mathbb{Z}$ so large that, for all $n \geq p$, we have $\xi(i, n) \notin C$ and $\xi(i, n) \notin X_{j}$ for all $j \in J$.

Define a sequence $\left(d_{r}\right)_{r \in \omega}$ of elements of $D$ by starting with $d_{0}$ and then inductively using the divisibility of $D / C$ to set $d_{r}=2 \cdot d_{r+1}+c_{r}$ with $d_{r+1} \in D$ and $c_{r} \in C$.

We claim that (the normal form of) $d_{r}$ contains $g(i, q+r)$ with coefficient $a$. We chose $a$ to make this true for $r=0$. If it is true for $r$, then, as $g(i, n)$ does not occur in $c_{r}$ for any $n$ (as $i$ is not a $C$-index), $g(i, q+r)$ occurs with coefficient $a$ in $d_{r}-c_{r}=2 \cdot d_{r+1}$. By the second consequence of the algorithm for converting expressions to normal form, it follows that $g(i, q+r+1)$ occurs with coefficient $a$ in the normal form of $d_{r+1}$, as desired.

Again referring to the algorithm for computing normal forms, we see that the set $J$, defined above using $d_{0}$, would be unchanged if we used any $d_{r}$ instead of $d_{0}$ in clause (i). Indeed, if some $j$ satisfied clause (i) for one of $d_{r}$ and $d_{r+1}$ but not for the other, then the equation $d_{r}=2 \cdot d_{r+1}+c_{r}$ would require some $g(j, m)$ to occur in $c_{r}$, so $j$ would be a $C$-index and would thus fail to satisfy clause (iii).

In view of the observations in the preceding two paragraphs, any final segment of the sequence $\left(d_{r}\right)$ is again a sequence of the same sort, only with a larger value of $q$. Since $p$ can obviously also be replaced by any larger number, we assume without loss of generality that $p=q$. Thus, henceforth, $d_{r}$ has $g(i, p+r)$ in its normal form, with coefficient $a \in R^{*}$.

The definition of the $d$ sequence gives us that

$$
\begin{aligned}
d_{0} & =2 d_{1}+c_{0} \\
& =4 d_{2}+2 c_{1}+c_{0} \\
& =\ldots
\end{aligned}
$$


for any $r$. Fixing some $r \geq 1$, let us consider the normal form of $d_{0}$ as obtained from the last line in the preceding display by first writing $d_{r}$ and $c=2^{r-1} c_{r-1}+\cdots+$ $2 c_{1}+c_{0}$ in normal form, then combining these normal forms to make an expression for $d_{0}=2^{r} d_{r}+c$, and finally normalizing the result. More precisely, let us consider the coefficient $s \in R$ of $\xi(i, p+r-1)$ in the resulting normal form, and in fact let us consider $s$ modulo $2^{r}$.

First, let us consider possible occurrences of $\xi(i, p+r-1)$ in the first expression for $2^{r} d_{r}+c$ that we got by combining normal forms of $d_{r}$ and $c$ (before normalizing the result). Any ordinal $\alpha$ that occurs in the normal form of $c \in C$ is itself in $C$ (closure condition (2)), and hence is different from $\xi(i, p+r-1$ ) by our choice of $p$. There may be occurrences of $\xi(i, p+r-1)$ in $d_{r}$, but these will have their coefficients multiplied by $2^{r}$ in the expression for $2^{r} d_{r}+c$, and therefore will not contribute to the coefficient $s$ modulo $2^{r}$.

It remains to consider occurrences of $\xi(i, p+r-1)$ that arise during the normalization of $2^{r} d_{r}+c$. These can arise as $\xi(j, n)$ during the normalization of $g(j, m)$ terms. (Recall that $\xi$ 's with different arguments can be equal, so we cannot a priori exclude cases with $j \neq i$. The rest of this paragraph shows that these cases can, nevertheless, be excluded.) Consider first those $g(j, m)$ for which $j$ is a $C$-index. That includes all the $g$ 's in the normal form of $c$ and possibly some in the normal form of $d_{r}$ as well. Any $\xi(j, n)$ arising during the normalization of these $g$ 's is in $C$ by closure condition (4) on $C$. These $\xi(j, n)$ 's therefore differ from $\xi(i, p+r-1)$ by our choice of $p$. It remains to consider those $g(j, m)$ in the normal form of $d_{r}$ that are not $C$-indices. One of these has $j=i$, which we treat in the next paragraph. The rest have $j \in J$. Any $\xi$ produced by their normalization is therefore in $X_{j}$ with $j \in J$ and is therefore different from $\xi(i, p+r-1)$ by our choice of $p$.

We have seen that the coefficient of $s$ modulo $2^{r}$ must arise entirely from the normalization of the term $2^{r} a g(i, p+r)$ in $2^{r} d_{r}+c$. This normalization process reads

$$
\begin{aligned}
2^{r} a g(i, p & +r)=2^{r-1} a g(i, p+r-1)-2^{r-1} a \xi(i, p+r-1) \\
& =2^{r-2} a g(i, p+r-2)-2^{r-2} a \xi(i, p+r-2)-2^{r-1} a \xi(i, p+r-1) \\
& =\ldots \\
& =a g(i, p)-a \xi(i, p)-2 a \xi(i, p+1)-\cdots-2^{r-1} a \xi(i, p+r-1) .
\end{aligned}
$$

So we see that the coefficient of $\xi(i, p+r-1)$ is $2^{r-1} a$. Since $a \in R^{*}$, this coefficient is not zero modulo $2^{r}$.

This proves that the coefficient of $\xi(i, p+r-1)$ in the normal form of $d_{0}$ is not divisible by $2^{r}$ and, in particular, is not zero. But $r$ was an arbitrary integer $\geq 1$, so the normal form of $d_{0}$ contains infinitely many non-zero terms. That is absurd, and so the lemma is proved.

It follows from the lemma just proved that the image of the projection of $D$ to $G / K$ is countable, for it is generated as an $R$-module by $\bar{g}(i, n)$ 's where $i$ is relevant and $n \in \mathbb{Z}$. That image is $D /(D \cap K)$, so to complete the proof that $D$ is countable and thus the proof of the theorem, it suffices to show that the kernel $D \cap K$ of that projection is also countable. We remarked earlier that $K$, being a free $R$-module, is fully starred, so we need only prove that $(D \cap K) /(C \cap K)$ is divisible. This we 
Consider an arbitrary element $d$ of $D \cap K$. As $D / C$ is divisible, we have $d=2 y-c$ for some $y \in D$ and some $c \in C$. Let their normal forms be

$$
\begin{aligned}
& c=\sum_{i} r_{i} g\left(i, n_{i}\right)+k_{c} \\
& y=\sum_{i} r_{i} g\left(i, n_{i}+1\right)+k_{y},
\end{aligned}
$$

where $k_{c}, k_{y} \in K$ and where the $g$ terms match except for a shift of $n$ 's because $c-2 y \in K$. (See the second consequence of the normalization algorithm.) Thus,

$$
\begin{aligned}
d=2 y-c & =\sum_{i} r_{i}\left[2 g\left(i, n_{i}+1\right)-g\left(i, n_{i}\right)\right]+2 k_{y}-k_{c} \\
& =-\sum_{i} \xi\left(i, n_{i}\right)+2 k_{y}-k_{c} .
\end{aligned}
$$

As $c \in C$, all the $g\left(i, n_{i}\right)$ that occur here are in $C$, and therefore so are all the $\xi\left(i, n_{i}\right)$ (cf. (2) and (4) in the closure conditions for $C$ ) as well as $k_{c}$ (by closure condition (2)). Furthermore, by closure condition (3), $C$ also contains all the terms $g\left(i, n_{i}+1\right)$, so $y-k_{y} \in C \subseteq D$. But also $y \in D$, so we conclude $k_{y} \in D$. Thus, the last displayed equation above exhibits $d$ as 2 times an element $k_{y}$ of $D \cap K$ plus terms from $C \cap K$. This completes the proof that $(D \cap K) /(C \cap K)$ is divisible and thus the proof of the theorem.

\section{REFERENCES}

1. M. Burke and M. Magidor, Shelah's pcf theory and its applications, Ann. Pure Appl. Logic 50 (1990), 207-254.

2. P. J. Cohen, The independence of the continuum hypothesis, Proc. Nat. Acad. Sci. U.S.A. 50 (1963), 1143-1148.

3. A. Dodd and R. B. Jensen, The core model, Ann. Math. Logic 20 (1981), 43-75.

4. A. Dodd and R. B. Jensen, The covering lemma for K, Ann. Math. Logic 22 (1982), 1-30.

5. R. B. Jensen, The fine structure of the constructible hierarchy, Ann. Math. Logic 4 (1972), 229-308.

6. W. Just, A. R. D. Mathias, K. Prikry, and P. Simon, On the existence of large p-ideals, J. Symbolic Logic 55 (1990), 457-465.

7. J.-P. Levinski, M. Magidor, and S. Shelah, Chang's conjecture for $\aleph_{\omega}$, Israel J. Math 69 (1990), 161-172.

8. W. Mitchell, The core model for sequences of measures, I, Math. Proc. Cambridge Phil. Soc. 95 (1984), 229-260.

9. R. M. Solovay, $2^{\aleph_{0}}$ can be anything it ought to be, The Theory of Models (J. W. Addison, L. Henkin, and A. Tarski, eds.), North-Holland, 1964, pp. 435.

10. E. Specker, Additive Gruppen von Folgen ganzer Zahlen, Portugal. Math. 9 (1950), 131-140.

Mathematics Dept., University of Michigan, Ann Arbor, Mi 48109, U.S.A.

E-mail address: ablass@umich.edu 